\title{
Combined liver resection and radiofrequency ablation for bilobar multiple colorectal liver metastasis
}

\author{
Jeong-Ik PARK* \\ Department of Surgery, Ulsan University Hospital, Ulsan, Korea
}

Lecture: Surgery is the gold standard for treatment in patients with resectable colorectal liver metastasis (CRLM), with reported 5 -year survival rates ranging from $40 \%$ to $60 \%$. Unfortunately, only $10 \%-25 \%$ of patients with CRLM are considered eligible for liver resection (LR). In others, extensive tumor burden within the liver or poor anatomical position of the tumors close to critical vascular or biliary structures precludes resection. In these patients, systemic therapy is offered with the goal of improving survival or potentially converting patients into surgical resection candidates. Although the outcome of systemic treatment is still being improved, the realistic goal of systemic treatment remains palliative. It is for this reason that more aggressive local therapeutic approaches are being pursued in patients with unresectable CRLM.

Over recent decades, many efforts have been made to increase the potentially resectable pool of patients with CRLM. The advent of more effective chemotherapy, specific techniques such as portal vein embolization, local ablation therapy, and two-stage hepatectomy have expanded the indications for surgery in patients with CRLM, and have offered a substantial survival benefit to selected patients. Although radiofrequency ablation (RFA) was initially used as a safe, well tolerated, easily repeated and less invasive technique in patients with unresectable CRLM, its indication has been expanded to those with resectable CRLM. However, unlike in HCC, the therapeutic efficacy of RFA in CRLM remains controversial, especially in patients with resectable tumors. Numerous studies have reported that RFA was inferior to LR in the treatment of CRLM, in terms of both local control and survival. In essence, there is little evidence to support the routine use of RFA in the management of CRLM, even in combination with LR [1,2].

\section{(1) Role of RFA in resectable CRLM}

At present, no prospective randomized trials have been reported. However, there are some retrospective reports of large numbers of patients regarding RFA versus LR for CRLM. Some reported that patients who received RFA had survival rates comparable to surgical group while others found that survival rates were better among patients undergoing LR. These benefits have led to the question of whether RFA could be used in the treatment of resectable patients as well, and whether the oncologic outcomes would be equivalent. Further studies are necessary to determine the efficacy of RFA versus LR.

The COLLISION trial is an ongoing phase III randomized trial comparing RFA and LR on patients with small $(<3 \mathrm{~cm})$ CRLM. Another ongoing trial, the LAVA trial, has also been designed to compare LR and RFA for the patients with CRLM. These new randomized trials might provide more evidence for the present equivalent outcome $[3,4]$.

\section{(2) Role of RFA in unresectable CRLM}

Systemic chemotherapy is the standard of care for patients with unresectable CRLM. However, liver metastatic lesions typically respond poorly to chemotherapy alone; thus, survival outcomes are suboptimal despite the recent introduction of novel chemotherapeutic agents and targeted therapies.

Several clinical studies demonstrated that RFA is an effective and safe treatment for CRLM; however, local and distant recurrent rates remain high. The combination of RFA and systemic chemotherapy has also been evaluated in some clinical studies and resulted in promising outcomes for CRLM as demonstrated by progression-free survival (PFS) and overall survival (OS). However, the treatment 
outcome of treated patients is still heterogenous. To deliver evidence on the beneficial effect of such an aggressive approach, RCT was required. To date, one randomized control trial has investigated the use of RFA for CRLM: A European intergroup randomized phase III study (European Organization for Research and Treatment of Cancer 40004 CLOCC trial). This trial randomized 119 patients with unresectable CRLM to receive either systemic chemotherapy alone $(n=59)$ or systemic chemotherapy plus RFA with or without LR $(\mathrm{n}=60)$. At the time of primary analysis with a median follow-up time of 4.4 years, the median OS was 45.3 (95\% confidence interval [CI], 33.1-NA) months for RFA combined with chemotherapy and 40.5 (95\% CI, 29.5-50.1) months for chemotherapy alone (hazard ratio $[\mathrm{HR}]=0.74 ; 95 \% \mathrm{CI}, 0.46-1.19 ; p=0.22$ ). Median PFS was 16.8 months $(95 \% \mathrm{CI}, 11.7-22.1)$ in the group assigned to combination treatment and 9.9 months $(95 \% \mathrm{CI}, 9.3-13.7)$ in the group assigned to chemotherapy alone $(\mathrm{HR}=0.63 ; 95 \% \mathrm{CI}, 0.42-0.95 ; p=0.025)$ [5]. After an extended follow-up of 9.7 years, the median OS was 45.6 months (95\% CI $=30.3-67.8$ months) for the RFA + chemotherapy arm and 40.5 months (95\% CI, 27.5-47.7 months) for the chemotherapy alone arm (HR $=0.58 ; 95 \% \mathrm{CI}=0.38-0.88 ; p=0.01)$. As previously reported, PFS was statistically significantly prolonged in the combined RFA with chemotherapy arm as compared with the chemotherapy alone $\operatorname{arm}(\mathrm{HR}=0.57 ; 95 \% \mathrm{CI}, 0.38-0.85 ; p=0.005)$ [6].

These data strongly suggest that the combined modality of aggressive local tumor treatment in combination with systemic chemotherapy can change the outcome of these patients considerably with a clear benefit on overall survival.

\section{(3) Combined intraoperative RFA and LR}

Patients who have multiple bilobar CRLM may be candidates for a two-stage approach. Another therapeutic option for patients with multiple CRLM can involve the combination of LR and RFA. RFA and LR should not be mutually exclusive. However, most published data on CRLM and RFA have focused on outcomes comparing patients who exclusively underwent either LR versus RFA alone. The data on combining LR with RFA for the treatment of multiple CRLM have been more limited.

Some comparative studies demonstrated that combination of RFA and LR provided worse survival compared to LR alone. Others showed comparable survival compared to LR alone. Until now the outcome of treatment in the literature are very heterogenous [7,8].

\section{Conclusion}

The evidence so far in the literature has shown that RFA is insufficient to be recommended as a radical oncological treatment of CRLM. However, RFA has established its position in patients with unresectable but ablatable CRLM with EORTC-CLOCC trial. When complete resection cannot be achieved, selective use of RFA can provide a modest survival benefit over chemotherapy alone. Combination of RFA and LR can be considered as a viable option to expand resectable pool in selected patients with multiple bilobar CRLM.

\section{REFERENCES}

1. Takahashi H, Berber E. Role of thermal ablation in the management of colorectal liver metastasis. Hepatobiliary Surg Nutr 2020;9:49-58.

2. Minami Y, Kudo M. Radiofrequency ablation of liver metastases from colorectal cancer: a literature review. Gut Liver 2013;7:1-6.

3. Puijk RS, Ruarus AH, Vroomen LGPH, van Tilborg AAJM, Scheffer HJ, Nielsen K, et al.; COLLISION Trial Group. Colorectal liver metastases: surgery versus thermal ablation (COLLISION) - a phase III single-blind prospective randomized controlled trial. BMC Cancer 2018;18:821.

4. Gurusamy K, Corrigan N, Croft J, Twiddy M, Morris S, Woodward N, et al. Liver resection surgery versus thermal ablation for colorectal LiVer MetAstases (LAVA): study protocol for a randomised controlled trial. Trials 2018;19:105.

5. Ruers T, Punt C, Van Coevorden F, Pierie JPEN, Borel-Rinkes I, Ledermann JA, et al. Radiofrequency ablation combined with systemic treatment versus systemic treatment alone in patients with non-resectable colorectal liver metastases: a randomized EORTC Intergroup phase II study (EORTC 40004). Ann Oncol 2012;23:2619-2626.

6. Ruers T, Van Coevorden F, Punt CJ, Pierie JE, Borel-Rinkes I, Ledermann JA, et al. Local treatment of unresectable colorectal liver metastases: results of a randomized phase II trial. J Natl Cancer Inst 2017;109:djx015.

7. Sasaki K, Margonis GA, Andreatos N, Kim Y, Wilson A, Gani F, et al. Combined resection and RFA in colorectal liver metastases: stratification of long-term outcomes. J Surg Res 2016;206:182-189.

8. Masuda T, Margonis GA, Andreatos N, Wang J, Warner S, Mirza MB, et al. Combined hepatic resection and radio-frequency ablation for patients with colorectal cancer liver metastasis: a viable option for patients with a large number of tumors. Anticancer Res 2018;38:6353-6360. 